\title{
Efecto de la fertirrigación en un cultivo de tomate sobre la sostenibilidad agrícola de un valle costero del desierto de Atacama, Chile
}

\author{
Effect of fertigation in a tomato crop on the agricultural sustainability \\ of a coastal valley of Atacama desert, Chile \\ Irene Bravo, Rodrigo Yucra y Pilar Mazuela ${ }^{1 *}$
}

\begin{abstract}
RESUMEN
Los valles costeros de la Región de Arica y Parinacota presentan condiciones climáticas excepcionales para el cultivo de hortalizas durante todo el año, siendo el principal proveedor de hortalizas para el país durante el invierno. La producción media regional de tomates supera significativamente la media nacional, con un alto valor comercial por la condición de "primor" que hace de su cultivo una actividad muy competitiva a nivel nacional. Dentro del proceso de producción, existen algunos aspectos de manejo del cultivo que pueden ser superados de manera de dar sustentabilidad económica y medioambiental a esta actividad al mejorar las técnicas de fertilización del tomate. El objetivo de este trabajo fue describir el manejo del fertirriego comúnmente utilizado en un invernadero comercial del valle de Azapa en sistemas de cultivo sin suelo y sugerir los aportes de fertilizantes para mejorar la nutrición del cultivo. El manejo racional del fertirriego, considerando los aportes de nutrientes que hace el agua de riego es una herramienta clave para la sustentabilidad de un cultivo de tomate. Los aportes nutricionales equilibrados evitan el envejecimiento prematuro de la planta con lo cual se mantiene la producción y calidad de tomate.
\end{abstract}

Palabras clave: sustratos, contraestación, sulfatos, calcio, disolución nutritiva, rendimiento.

\begin{abstract}
The coastal valleys of the Region Arica y Parinacota (Chile) have exceptional climatic conditions for vegetable crops during the entire year; they are the main provider of vegetables in the country in winter. The mean regional production of tomatoes is greater than the national mean, with high commercial value due to the "prime" condition that makes its growing a very competitive activity in the country. Some aspects of crop management during the production process may be improved in order to provide economic and environmental sustainability to this activity, especially improvement of the techniques of tomato fertigation. The objective of this study was to describe the fertigation management commonly used in a commercial greenhouse by soilless culture in the Azapa valley and to suggest fertilizer inputs to improve crop nutrition. The rational management of fertigation, considering that nutrient input along with the irrigation water is a key tool for the sustainability of a tomato crop. Equilibrated nutritional input avoids premature aging of the plant, which maintains the production and quality of the tomatoes.
\end{abstract}

Key words: substrates, off-season, sulfates, calcium, dissolution, yield.

\section{Introducción}

La región de Arica y Parinacota presenta condiciones climáticas excepcionales para el cultivo de hortalizas durante todo el año, siendo el principal proveedor de hortalizas para el país durante el invierno (Saavedra y Tapia, 2009). La hortaliza de mayor valor económico por superficie cultivada, uso intensivo de insumos y mano de obra es el tomate para consumo fresco. Además, la media regional supera en 59\% la media nacional y en $20 \%$ a la media de la región de Valparaíso (Tabla 1). El valle de Azapa se sitúa en las coordenadas 18³1'2"S $70^{\circ} 11^{\prime} 31$ 'O. El ancho del valle es variable y fluctúa entre $700 \mathrm{~m}$ a $2.200 \mathrm{~m}$; con una pendiente de $1,7 \%$ y un largo aproximado de $58 \mathrm{~km}$. Este valle cuenta

\footnotetext{
1 Departamento de Producción Agrícola, Universidad de Tarapacá, Arica, Chile

* Autor correspondiente: pmazuela@uta.cl
}

Fecha de Recepción: 9 Septiembre, 2016.

Fecha de Aceptación: 25 Noviembre, 2016.

DOI: $10.4067 / \mathrm{S} 0718-34292016005000031$. 
Tabla 1. Superficie total y rendimiento medio de tomate para consumo, según datos nacional y por región.

\begin{tabular}{lcc}
\hline Región & ${ }^{1}$ Superficie (há) & 2Rendimiento $\left(\mathrm{kg} \mathrm{m}^{-2}\right)$ \\
\hline Nacional & 6.309 & 7,11 \\
XV & 840 & 11,29 \\
III & 212 & 6,16 \\
IV & 358 & 3,08 \\
V & 1.179 & 9,44 \\
VI & 1.062 & 5,87 \\
VII & 938 & 6,89 \\
VIII & 467 & 4,97 \\
RM & 1.080 & 6,19 \\
\hline
\end{tabular}

Fuente: ${ }^{1}$ INE, 2008, ${ }^{2}$ INE, 2010.

con condiciones edafoclimáticas que le permiten el cultivo del tomate durante todo el año. Predominan las condiciones de clima de desierto costero con nubosidad abundante, ausencia de heladas, vientos moderados, con alta humedad relativa y alta radiación solar directa, durante todo el año. La temperatura media anual es de $18{ }^{\circ} \mathrm{C}$, las máximas medias anuales de $23,6^{\circ} \mathrm{C}$ y mínimas de $13,8^{\circ} \mathrm{C}$ (Torres y Acevedo, 2008). El cultivo de tomate se hace bajo malla como método de protección de cultivos para disminuir la incidencia de plagas y enfermedades en las plantas. Riquelme-Garcés et al. (2013), indica que la producción bajo malla y al aire libre es de 20,6 y 7,6 $\mathrm{kg} \mathrm{m}^{-2}$, respectivamente. En el mismo trabajo se indica que la eficiencia hídrica es de $25,6 \mathrm{~kg}$ de tomate por $\mathrm{m}^{3}$ de agua de riego aportado en cultivo bajo malla y de solo $12,5 \mathrm{~kg} \mathrm{~m}^{-3}$, en cultivos al aire libre. La mayor producción de tomate bajo malla se debe a las mejores condiciones de cultivo que evita el envejecimiento prematuro de la planta y aumenta el calendario de producción comercial, por tanto, aumenta la producción total por superficie cultivada.

La demanda de los consumidores por productos de calidad han generado cambios en el manejo de cultivos para optimizar la producción y calidad de las hortalizas. Estos no solamente demandan productos de calidad sino, además, valoran que los procesos de producción sean sustentables. $\mathrm{La}$ sostenibilidad o sustentabilidad agrícola es un término para expresar la necesidad de generar condiciones que permitan que esta actividad pueda permanecer durante el tiempo.

Un estudio liderado por Espina (1971) advierte sobre los factores de manejo que más inciden en la productividad del cultivo de tomate en el valle de Azapa, siendo los más importantes el control fitosanitario del suelo, el manejo del agua de riego (métodos de riego) y la aplicación de fertilizantes. Para el control fitosanitario del suelo, muchos agricultores del valle de Azapa están cultivando en sustrato. Respecto a la aplicación de fertilizantes, se indica que hay un desconocimiento generalizado de las normas técnicas elementales de dosificación, situación que persiste hasta hoy (González-Vallejos et al., 2013). Diversos autores han desarrollado recomendaciones de referencia para el fertirriego de un cultivo de tomate (Tabla 2). El manejo de la fertirrigación incide en la sustentabilidad de los cultivos intensivos por la salinización de suelo y aguas; en el aumento en los costos de producción por ineficiencia en el uso de los fertilizantes y, en el envejecimiento prematuro de la planta que afecta su producción y calidad de fruto.

El objetivo de este trabajo fue describir el manejo del fertirriego comúnmente utilizado en un invernadero comercial del valle de Azapa en sistema de cultivo sin suelo y sugerir los aportes de fertilizantes para mejorar la nutrición del cultivo.

\section{Material y Método}

\section{Localización y descripción general}

El sector en el que se realizó el ensayo está localizado en las dependencias de un predio comercial, ubicado en el kilómetro 3 del valle de

Tabla 2. Niveles de referencia para la disolución nutritiva para un cultivo de tomate, según autor.

\begin{tabular}{lcccccrc}
\hline \multirow{2}{*}{ Autor } & \multicolumn{3}{c}{$\mathrm{me} \mathrm{L}^{-1}$} \\
\cline { 2 - 7 } & $\mathrm{NO}_{3}^{-}$ & $\mathrm{H}_{2} \mathrm{PO}_{4}^{-}$ & $\mathrm{SO}_{4}{ }^{2-}$ & $\mathrm{NH}_{4}^{+}$ & $\mathrm{K}^{+}$ & $\mathrm{Ca}^{2+}$ & $\mathrm{Mg}^{2+}$ \\
\hline Sonneveld, 1980 & 10,5 & 1,5 & 5,0 & 0,5 & 7,0 & 7,5 & 2,0 \\
García y Urrestarazu, 1999 & 12,5 & 2,0 & 3,5 & & 5,0 & 10,0 & 3,6 \\
Urrestarazu et al., 2005 & 13,0 & 1,75 & 2,5 & 1,0 & 7,5 & 8,0 & 2,5 \\
\hline
\end{tabular}


Azapa, Región de Arica y Parinacota. El ciclo de cultivo fue otoño-primavera, durante los meses de mayo-diciembre de 2015 con un total de 245 días. El ensayo se estableció en una estructura de invernadero de tipo plano con pendiente, en una superficie de $7.828 \mathrm{~m}^{2}$ con una cubierta de malla antiáfido.

\section{Manejo del cultivo}

Se utilizaron plantas injertadas 7742 sobre Maxifort. Se colocaron tres plantas de tomate a doble eje en un contenedor de fibra de coco, en sistema de cultivo sin suelo. El marco de plantación fue de 0,4 $\mathrm{m} \times 2 \mathrm{~m}$. Sobre cada saco de cultivo se colocaron cuatro goteros autocompensados de $2 \mathrm{~L} \mathrm{hr}^{-1}$.

\section{Agua de riego y aporte de fertilizantes}

En la Tabla 3 se indica la media de las características químicas del agua de riego y los aportes en fertilizantes según las indicaciones del asesor que comúnmente recomienda en el valle de Azapa.

\section{Parámetros de fertirriego y producción}

Para evaluar los parámetros de fertirriego se colocaron dos goteros control y dos bandejas de drenaje distribuidos en sectores representativos al interior del invernadero. Dos veces por semana se midió conductividad eléctrica $\left(\mathrm{dSm}^{-1}\right), \mathrm{pH}$, aporte hídrico y porcentaje de drenaje. Con estos datos se obtuvo el consumo hídrico $\left(\mathrm{L} \mathrm{m}^{-2}\right)$ y eficiencia hídrica del cultivo ( $\mathrm{kg}$ producidos por $\mathrm{m}^{3}$ aportado).

\section{Discusión de Resultados}

Los resultados de la disolución nutritiva resultante se indican en la Tabla 3 y los datos de producción, consumo hídrico y eficiencia hídrica se observan en la Tabla 4.

Uno de los problemas descritos por Espina (1971) es el desconocimiento de las normas técnicas elementales de dosificación, más aun si se fertiliza por medio del agua de riego. En primer lugar, se observa que no se consideran las disoluciones de referencia desarrolladas por diversos autores para el cultivo de tomate (Tabla 2) ni los aportes de aniones y cationes en el agua de riego (Tabla 3 ). En consecuencia, la disolución nutritiva resultante tiene un exceso de sulfatos y un déficit de calcio, principalmente. Una de las principales precauciones al momento de formular una disolución nutritiva es evitar juntar calcio con sulfato para soslayar la precipitación del calcio, por lo tanto, no se recomienda eliminar los fertilizantes sulfatados y aumentar el nitrato cálcico en dosis de uno a cuatro $\mathrm{me}^{-1}$, según la disolución de referencia con la que se desee trabajar. La disolución nutritiva equilibrada aumenta la vida útil de la planta y disminuye la emisión de iones al medio ambiente que en el caso del cultivo

Tabla 3. Media del aporte de aniones y cationes del agua de riego (AR) del canal Lauca, media de aporte por fertilizantes (AF) y disolución nutritiva resultante (DNR) para un cultivo de tomate en un predio comercial del valle de Azapa, en cultivo sin suelo.

\begin{tabular}{lccccccccc}
\hline & \multicolumn{1}{c}{$\mathrm{me} \mathrm{L}{ }^{-1}$} \\
\cline { 2 - 9 } & $\mathrm{NO}_{3}{ }^{-}$ & $\mathrm{H}_{2} \mathrm{PO}_{4}^{-}$ & $\mathrm{SO}_{4}{ }^{-2}$ & $\mathrm{Cl}^{-}$ & $\mathrm{NH}_{4}{ }^{+}$ & $\mathrm{Ca}^{+2}$ & $\mathrm{~K}^{+}$ & $\mathrm{Mg}^{2+}$ & $\mathrm{Na}^{+}$ \\
\hline $\mathrm{AR}$ & 0,3 & 0,0 & 3,7 & 2,4 & 0,0 & 2,8 & 0,2 & 1,6 & 3,3 \\
$\mathrm{AF}$ & 8,7 & 2,0 & 6,0 & 0,0 & 1,0 & 4,0 & 8,3 & 3,4 & 0,0 \\
$\mathrm{DNR}$ & 9,0 & 2,0 & 9,7 & 2,4 & 1,0 & 6,8 & 8,5 & 5,0 & 3,3 \\
\hline
\end{tabular}

Tabla 4. Valores medios de conductividad eléctrica (CE), pH, producción, consumo hídrico y eficiencia en el uso del agua para un cultivo de tomate en un predio comercial del valle de Azapa, en cultivo sin suelo.

\begin{tabular}{lr} 
CE disolución nutritiva $\left(\mathrm{dS} \mathrm{m}^{-1}\right)$ & 2,94 \\
$\mathrm{pH}$ disolución nutritiva & 5,7 \\
Producción $\left(\mathrm{kg} \mathrm{m}^{-2}\right)$ & 13,3 \\
Consumo hídrico $\left(\mathrm{L} \mathrm{m}^{-2}\right)$ & $1.471,5$ \\
Eficiencia en uso del agua (kg producidos m${ }^{-3}$ agua aportado) & 9,0 \\
\hline
\end{tabular}


del tomate en el valle de Azapa, genera 8,82 $\mathrm{T} \mathrm{ha}^{-1}$ año ${ }^{-1}$ (Riquelme-Garcés et al., 2013).

Al comparar el rendimiento de tomate, este es superior a la media regional y casi duplica la media nacional (INE, 2010). Sin embargo, Riquelme-Garcés et al. (2013), indica que la media productiva de un cultivo de tomate para consumo en fresco en el valle de Azapa, bajo malla, es de $20,6 \mathrm{~kg} \mathrm{~m}^{-2}$, un $55 \%$ superior al obtenido en este trabajo. Respecto a la eficiencia en el uso del agua, el resultado es inferior al obtenido por RiquelmeGarcés et al. (2013), de 25,6 $\mathrm{kg} \mathrm{m}^{-3}$ y menor a los $31,9 \mathrm{~kg} \mathrm{~m}^{-3}$, observados por Torres (1998). Esto podría explicarse por la frecuencia y tiempo de aplicación de riego donde no se consideran las características físicas del sustrato. Para producir un kilogramo de tomate se necesitaron $111 \mathrm{~L}$ de agua de riego. Riquelme-Garcés et al. (2013), necesitó $39 \mathrm{~L}$ de agua para producir un $\mathrm{kg}$ de tomate bajo malla y $80 \mathrm{~L} \mathrm{~kg}^{-1}$ al aire libre. Este último valor, muy similar a los observados por Villavicencio et al. (2013), donde indica una huella hídrica referencial de $89,1 \mathrm{~L} \mathrm{~kg}^{-1}$, en la zona alta de la cuenca del ríos San José; $69,4 \mathrm{~L} \mathrm{~kg}^{-1}$, en la zona media y 95,6 $\mathrm{L} \mathrm{kg}^{-1}$, en la zona baja, dando una media de $84,7 \mathrm{~L} \mathrm{~kg}^{-1}$, en la cuenca del río San José que constituye el valle de Azapa.

\section{Conclusión}

Los resultados de la descripción del manejo del fertirriego en un predio comercial de tomate para consumo fresco en el valle de Azapa indican que se pueden mejorar las labores culturales para dar sustentabilidad al cultivo. Se sugiere considerar las características del agua de riego en el cálculo de los aportes de fertilizantes en la disolución nutritiva, eliminar el aporte de fertilizantes sulfatados y aumentar la aplicación del nitrato de calcio. Se recomienda mejorar las técnicas de riego, considerando las características físicas del sustrato, en sistema de cultivo sin suelo. La sustentabilidad económica y medioambiental del cultivo del tomate puede mejorar al eliminar el aporte de nutrientes que están disponibles en el agua de riego y mejorando la eficiencia en el uso del agua.

\section{Agradecimientos}

Este trabajo ha sido financiado por el proyecto UTA Mayor 9720-14.

\section{Literatura Citada}

Espina, L.

1971. Estudio agroeconómico del valle de Azapa. Junta de Adelanto de Arica-Universidad de Chile, Santiago de Chile, $155 \mathrm{p}$.

García, M. y Urrestarazu, M.

1999. Recirculación de la disolución nutritiva en los invernaderos de la Europa del Sur. Caja Rural de Granada, España, 171 pp.

González-Vallejos, F.; Riquelme-Garcés, A.; Contreras-Luque,

P. y Mazuela, P.

2013. Antecedentes generales para la sustentabilidad de la producción hortícola en el valle de Azapa, Arica, Chile. Idesia, (31) 4: 119-123.

Instituto Nacional de Estadísticas

2010. Información Hortícola. Publicación Especial 2008-2009.

INE Ediciones, Santiago de Chile, 128 p.

Instituto Nacional de Estadísticas

2008. VII Censo Agropecuario y Forestal 2006-2007. Resultados preliminares. INE Ediciones, Santiago de Chile, 444 p.

Riquelme-Garcés, A.; González-Vallejos, F.; Contreras-Luque,

P.; Mazuela, P.

2013. Manejo del cultivo de hortalizas y su efecto en la sustentabilidad de un valle costero del desierto de Atacama, Chile. Idesia 31: (3) 113-117.

Saavedra, A. y Tapia, L.

2009. Evaluación de las estrategias de producción de tomate (Lycopersicon esculentum Mill) fuera de estación para la agricultura de la provincia de Arica, Chile, entre los años 1995-2005. Idesia, 27 (2): 91-96.

Sonneveld, C.

1980. Growing cucumbers and tomatoes in Rockwool. Proceedings Fifth International Congress on Soilless Culture. Wageningen, Netherlands, pp. 253-262

Torres, A.

1998. Programa de investigación en parcelas experimentales cuenca del río San José, Ministerio de Obras Públicas. Dirección General de Aguas, Departamento de Estudios y Proyectos, Universidad de Tarapacá, Instituto de Agronomía, SIT N $\mathrm{N}^{\circ} 52$.

Torres, A. y Acevedo, E.

2008. El problema de salinidad en los recursos suelo y agua que afectan el riego en los valles de Lluta y Azapa en el norte de Chile. Idesia, 26 (3): 31-44.

Urrestarazu, M.; Mazuela, P.; Del Castillo, J.; Sabada, S. y Muro, J. 2005.

Fibra de pino: un sustrato ecológico. Horticultura Internacional, 49: 28-33.

Villavicencio, A; Riquelme, P.; Pérez, A.

2013. Huella hídrica en tomate fresco, Pp. 87-92. En: Determinación de la huella del agua y estrategias de manejo de recursos hídricos. Osorio, A. (Ed.). Series Actas INIA 50. La Serena, Chile. 